\title{
Research on Design of the Elderly Kitchen Based on Behavioral Logic
}

\author{
Yushan Ding ${ }^{a}$, Jinglian Chen ${ }^{\text {b,* }}$ \\ College of Arts and Design, Beijing Forestry University, Beijing, China \\ a dingyushan913@163.com, bjinglianchen@hotmail.com \\ *corresponding author
}

Keywords: Elderly kitchen, Field visit, Level task decomposition, Action analysis

\begin{abstract}
In the rapid urbanization process, the overall cabinet brand is all-encompassing, but its functional design and consideration of details do not fully consider the needs of Chinese consumers, especially the elderly. This study explored the behavior of elderly users in the kitchen, and summarized kitchen design principles, and optimized the cooking experience. The application of the hierarchical task analysis method analyzed the operation behavior of elderly users in the kitchen, and the action economy principle improved the cooking action, and the reasons behind the action are sorted out which from three aspects: user environment, user operation and user expectation. Through the hierarchical task decomposition, action analysis and the principles of action economy, the specific design methods are summarized. Concrete kitchen design principles are generalized to guide the design practice and improve the kitchen experience for elderly users.
\end{abstract}

\section{Introduction}

China has entered an aging society in $2000^{[1]}$. Due to the cultural habits of Chinese society, the proportion of elderly people who choose to age in places mode accounts for a large proportion. As the age increases, the physiology and mental function of the elderly decline, and these factors often increase the likelihood of accidents in the home environment. The kitchen is a complex room, and work related to meal preparation will take place here and it will take a lot of time to complete. Elderly people are less sensitive to their surroundings than young people. Therefore, the working environment is particularly important for human factors. This study aims to establish a set of kitchen design principles that are safe and comfortable for the elderly in the country, so as to enhance the self-reliance of the elderly and their ability to master the living environment.

For the problem of the aging kitchen, most of the researches at home and abroad have been concentrated in the three aspects of layout planning, space size and specific functional area design from the perspective of "object". The "object" design focuses on the type, combination, structure, texture, size, etc. of the object, and lacks interaction with the users ${ }^{[2]}$. In the field of interactive design, Mr. Xin Xiangyang, the former president of the School of Design of Jiangnan University, put forward the concept of "behavior logic", and called "reasonable organizational behavior as the basis for decision-making" as "behavior logic" and "emphasis the decision-making basis for the rational allocation of its own attributes" is called "physical logic" [3]. Because the behavior of the elderly is influenced by various uncertain factors such as scene, emotion, man-made, and society, it is necessary to study the behavior characteristics of the elderly from the perspective of behavioral logic, and consider the design method of cabinets in order to improve the rationality and user experience of the elderly in the kitchen.

\section{Methods}

In the study of the whole kitchen cabinet, the researchers must first understand the user, and understand the real scene of the use of the kitchen, understand the user's views and opinions on the kitchen, observe the difference between the elderly group and the general group in the use of the kitchen, and avoid the designer falling into the design model centered on self-awareness. User 
observation aims to enable designers to better understand the needs of users by participating in people's daily lives, systematically providing design principles, and developing products that meet user and market needs. It is not to find some scientific or even mechanical logical connection of the behavior process itself, but to emphasize behavior as the main body of cabinet design, and consider the process rationality and user experience of the cabinet design according to the specific task.

\subsection{Field visit method}

The purpose is to understand the way users behave and their motivations. This method can provide information about the user's operating environment that other methods can't get. It can reveal how users define what is really valuable to them, and understand the user's perception of the kitchen.

\subsection{The principle of action economy.}

The basic idea of the principle of action economy is to develop operational methods based on the principle of minimizing worker fatigue and maximizing the efficiency of workers, and then equipped with effective processing tools, mechanical equipment and reasonable work place layout ${ }^{[4]}$. The Gilbreths conducted in-depth research in this field, created action research, and proposed the principles of action economy (22 principles). After further refinement by other scholars, four action economic principles were summarized: reducing the number of actions; Simultaneous action; shorten the movement distance; light movement. It also includes three aspects of improvement, namely human body use, work place layout and work tools and equipment. The principle of action economy effectively reduces labor intensity and greatly improves work efficiency. Action research and action economy principles have been widely used in production operations $^{[5]}$.

\section{Analysis of kitchen operations}

The author conducted a survey of five elderly households living in Haidian District, Beijing by field visits and interviews. The age of the survey was focused at the age of 65 , and the whole process of cooking was recorded. Table 1 shows the kitchen configuration of the surveyed residential buildings.

Table 1 The kitchen configuration of five residential buildings.

\begin{tabular}{cccccc}
\hline & Subject A & Subject B & Subject C & Subject D & Subject E \\
\hline $\begin{array}{c}\text { Kitchen area } \\
\left(\mathrm{m}^{2}\right)\end{array}$ & 2.24 & 3.4 & 4.16 & 3.4 & 3.18 \\
Cabinet type & $\mathrm{U}$ & $=$ & $=$ & $=$ & $=$ \\
\hline
\end{tabular}

\subsection{The hierarchical task analysis}

Task decomposition of the cooking behavior of the surveyed users (Figure 1), the entire task can be summarized as the use of the object (cabinet, kitchen utensils, ingredients) and how the user interacts with the object (cabinet, kitchenware, ingredients). The use environment is mainly focused on the working triangle of the kitchen, namely the sink area, the preparation area and the cooking area.

\subsection{Action analysis}

Further, the user's actions are divided into three categories, which are taken, placed, taken, thrown, and twisted into one type, cut, cut into one type, washed into one type, combing the elderly user's kitchen operation action (Figure 2).

The other four users are analyzed in turn. It is found that the distance between the cleaning action and the garbage disposal action is too far, and the distance of the moving line is increased. Take the items to the sink area for cleaning more times, increasing the distance of the moving line. More frequent pick and place actions. The position of the kitchen utensils is inconsistent, increasing 
the number of actions and so on (Table 2).

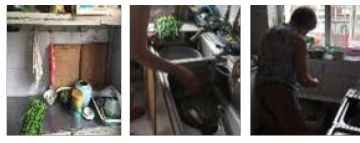

$\mathrm{T}_{1}$ : Take the leek and beans from the table.

$\mathrm{T}_{2}$ : Use scissors to assist in selecting vegetables.

$\mathrm{T}_{3}$ : Choose the dish on the drain board.

$\mathrm{T}_{4}$ : Put a wash basin in the sink and add aloe vera washing solution.

$\mathrm{T}_{5}$ : The garbage selected during the washing process is thrown into the trash can.

$\mathrm{T}_{6}$ : Put the dish soaked in the washing solution into another sink

$\mathrm{T}_{7}$ : Wash the vegetables with water and wash the dishes on the drain board.

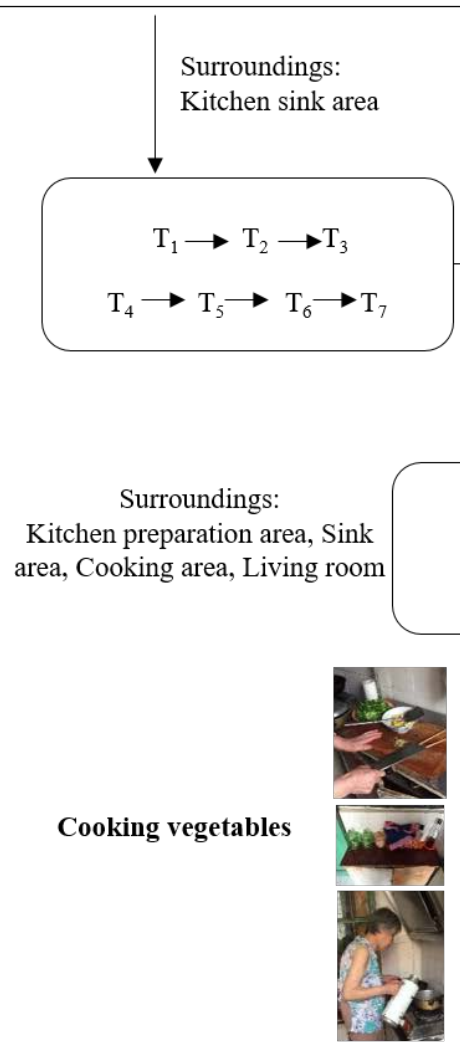

Preparing vegetables
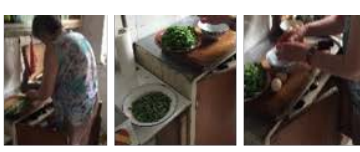

$\mathrm{T}_{1}$ : Take the chopping board out of the preparation area drawer and wipe it with a rag.

$\mathrm{T}_{2}$ : Take the knife out of the preparation area drawer and rinse it with water.

$\mathrm{T}_{3}$ : Chop vegetables.

$\mathrm{T}_{4}$ : Take out the plate on the countertop of the preparation area, place the cut vegetables, and place them to cooking area side.

$\mathrm{T}_{5}$ : Repeat the above steps to prepare amaranth.

$\mathrm{T}_{6}$ : Remove the eggs from the refrigerator and rinse with water.

$\mathrm{T}_{7}$ : Stir eggs.

$\mathrm{T}_{8}$ : Cut ginger.

Surroundings:

Kitchen preparation area,

Sink area, Living room

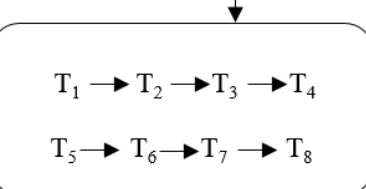

$\mathrm{T}_{1} \rightarrow \mathrm{T}_{2} \rightarrow \mathrm{T}_{3} \rightarrow \mathrm{T}_{4} \rightarrow \mathrm{T}_{5}$

$\mathrm{T}_{6} \rightarrow \mathrm{T}_{7} \rightarrow \mathrm{T}_{8} \rightarrow \mathrm{T}_{9} \rightarrow \mathrm{T}_{10}$

$\mathrm{T}_{1}$ : Open stove fire A

$\mathrm{T}_{2}$ : Rinse the wok and place it on the stove

$\mathrm{T}_{3}$ : Take the oil bottle from the mixing cabinet and pour it into the pot

$\mathrm{T}_{4}$ : Wait for oily heat, and add egg liquid

$\mathrm{T}_{5}$ : Rinse the shovel and stir the eggs

$\mathrm{T}_{6}$ : Adjust the firepower, take the bowl and hold the eggs, and place them on the chopping board.

$\mathrm{T}_{7}$ : Pour the oil, add the ginger, add the leeks, stir, add the eggs,

$\mathrm{T}_{8}$ : Take the salt shaker from the spice cabinet and take the appropriate amount into the pot.

$\mathrm{T}_{9}$ : Place the wok on the stove $\mathrm{B}$ and place the skillet on the stove A.

$\mathrm{T}_{10}$ : Take the kettle from the living room, put hot water in the skillet, and put the kettle back in the living room

$\mathrm{T}_{11}$ : Make another dish

Figure 1 Task decomposition of the cooking behavior of the surveyed users.

Table 2 Summary of action problems.

\begin{tabular}{|c|c|}
\hline Notation & Action description \\
\hline $1 \rightarrow$ (4) (2) $\rightarrow$ & $\begin{array}{l}\text { The cleaning action is too far away from the garbage disposal } \\
\text { action, increasing the distance of the moving line }\end{array}$ \\
\hline & $\begin{array}{l}\text { Take the items to the sink area for cleaning more times, } \\
\text { increasing the distance of the moving line }\end{array}$ \\
\hline & $\begin{array}{l}\text { More frequent pick and place actions. The position of the kitchen } \\
\text { utensils is inconsistent, increasing the number of actions }\end{array}$ \\
\hline
\end{tabular}

\subsection{Question Category}

According to the action analysis method, the reasons behind the action are sorted out from the user environment, user operation and user expectation, which is a bridge between identifying user needs and product development (Table 3). 
Table 3 Summary of the inconveniences and difficulties in the use of current kitchen for the participants.

\begin{tabular}{|c|c|}
\hline User perspective & Inconveniences and difficulties \\
\hline User environment & $\begin{array}{l}\text { - The sink area is too small. } \\
\text { - The sink area and the preparation area are not on the same side. } \\
\text { - Cooking area is too small. } \\
\text { - The seasoning placement area is too far away from the stove area. } \\
\text { and the lower cooking height makes it easy to put the elbows. } \\
\text { - The bottom floor of the cabinet. } \\
\text { - The cabinet door is easy to meet after opening. } \\
\text { left in the trash. }\end{array}$ \\
\hline User operation & $\begin{array}{l}\text { Poor memory leads to forgetting to turn off the fire. } \\
\text { - Arthritis causes difficulty in using the cabinet. } \\
\text { - One-handed use of pots to water to increase the difficulty of operation. } \\
\text { The cleaning area is used most frequently. } \\
\text { - Too much food in the refrigerator increases the difficulty of taking. } \\
\text { The chopping board and rolling pin are used more frequently. }\end{array}$ \\
\hline User expectation & $\begin{array}{l}\text { - The sink area is provided with a retractable combination sink, which can } \\
\text { adjust the sink space according to the size of the kitchenware and the } \\
\text { ingredients. } \\
\text { - The inside of the cabinet is designed as a plug-in structure, so that the } \\
\text { space can be freely set, so that kitchen utensils of different sizes can be } \\
\text { placed. } \\
\text { - the lowest floor cabinet. } \\
\text { - Lifting cabinet, hanging cabinet door to pull way. } \\
\text { - Countertop embedded trash can. } \\
\text { - Appliance timing function. } \\
\text { - Storage method of chopping board and rolling pin. } \\
\text { - Height adjustment of the countertop in the preparation area }\end{array}$ \\
\hline
\end{tabular}

\section{Kitchen optimization design based on behavioral logic}

Through hierarchical task decomposition, action analysis, and action economic principles, the reasons behind the behaviors are analyzed from the user environment, user operations, and user expectations, to bridge the gap between user needs and product development. Three design principles are summarized. Optimize the action mode. Reduce the difficulty of the action. Reduce the accident rate. According to the design principles, combined with the design purpose, a specific design method is proposed.

\subsection{Optimize the action mode}

From the perspective of interaction design, kitchen behavior can be understood as the interaction between the user and the kitchen, emphasizing the information exchange between the kitchen and the user. In the design process, it is necessary to fully consider the user's operating habits, usage patterns, etc., from the perspective of user behavior and needs to design the product. The design of well-old kitchen is to seek to support the communication and interaction between users and kitchen products and others in a specific context space, and to optimize the interaction between people and the kitchen and society. The sink area is provided with a rotatable structure that adjusts the angle of the sink according to the shape of the kitchenware and ingredients.

\subsection{Reduce the difficulty of the action}

Based on the scale of human-computer interaction, in the process of designing the elderly 
kitchen, the dimensions of the cabinets, racks, countertops, etc. should be reconsidered to meet the needs of the elderly in different obstacle types. The height of the countertop can be self-adjusting. The air pressure lifting device can be added under the countertop. The height of the countertop can be adjusted through the valve to adapt to the elderly with different heights. The interior of the cabinet is designed as a plug-in structure, so that the space can be freely set. In order to place kitchen utensils of different sizes, make full use of the internal space, reduce the difficulty of taking the ingredients in the cabinet. Remove the bottom floor cabinet to avoid the user bending or squatting greatly. The cabinet is added to the ball screw structure to realize the cabinet height adjustment.

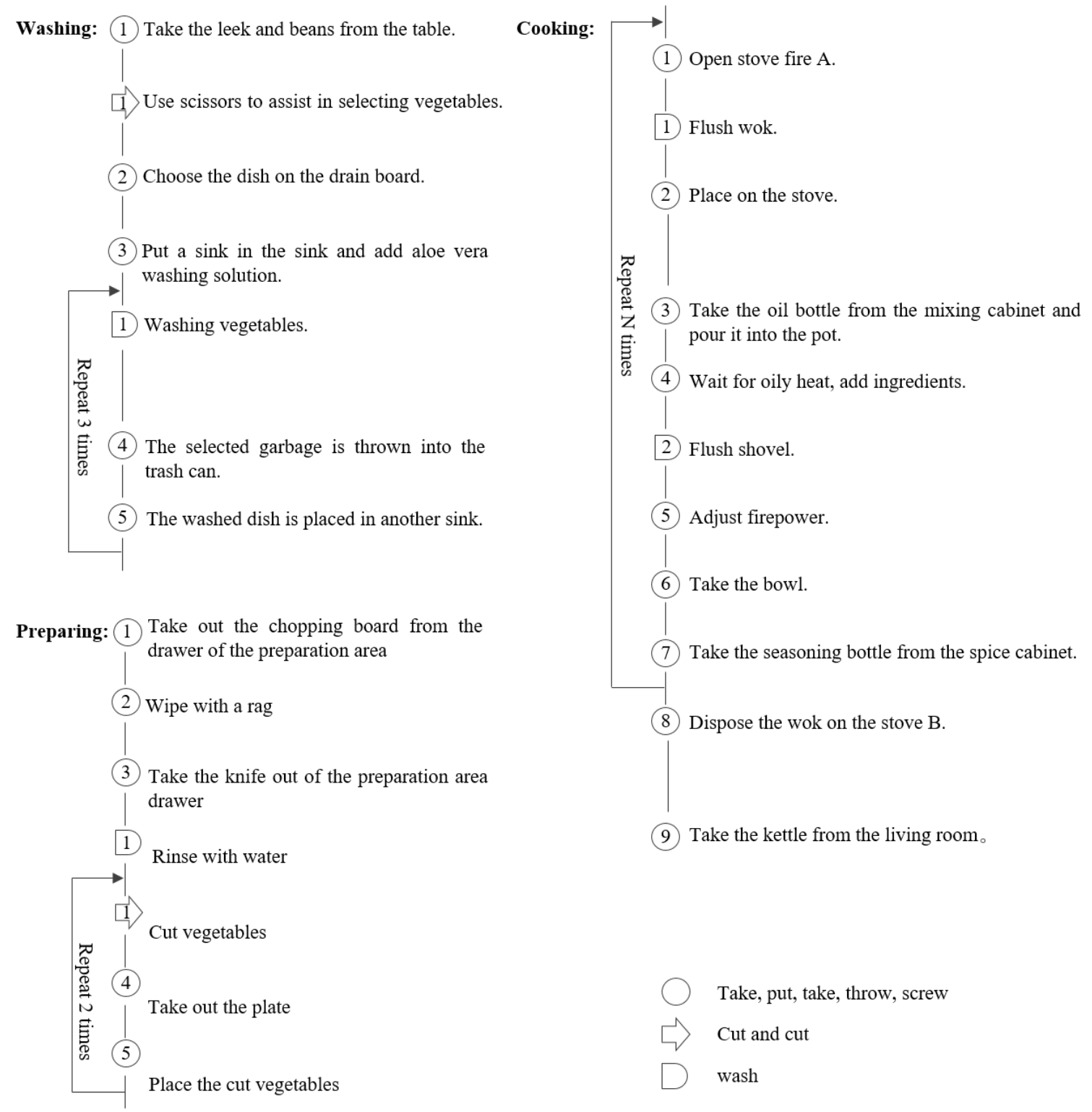

Figure 2 The elderly user's kitchen operation action analysis.

\subsection{Reduce the accident rate}

The living environment is most prone to fall accidents, in the bathroom, bedroom, stairs, kitchen, living room and other places. The occurrence of home accidents is a mistake in some aspects of a group of behaviors. The mistakes are mainly caused by unsafe environment, unsafe behavior and opportunity factors. The formation of unsafe behavior is affected by environmental factors such as inconvenient use, insufficient support, and personal factors. Behavior is generated by the interaction of individuals and the environment. The visual acuity of the elderly is reduced, and it is difficult to observe the size of the firepower. The table top can display the intensity of the halo to visually reflect the size of the firepower. The memory of the elderly is declining, and the timekeeping 
function is often forgotten, and the cooking time is reminded by setting the time. The garbage disposal slot is set in the countertop area of the preparation area, and the embedded trash can is added to reduce the chance of the kitchen floor slipping.

\section{Conclusion and recommendations}

Fusion of products and our lives will give better play to the role. The purpose of field visits is not just to discover what users are asking for, but to understand how people live and understand what changes they might want. Hierarchical task decomposition helps to sort out what is being observed in the field, so you can quickly discover useful user knowledge. The purpose of motion analysis is to optimize the cooking experience by analyzing and researching the movements of the user's parts during cooking, removing unnecessary movements, and designing suitable cabinet appliances and work place layouts.

One limitation of the present study should be acknowledged. The number of participants was relatively small and the data of the field visits might not be generalizable to all elderly people in China. Pursuing the core nature of the problem from the behavioral problems of the elderly, it is found that the kitchen environment, physiological state and psychological state are the three factors that affect the operating experience of the elderly kitchen. Designers can do further user research in terms of physiological state and mental state, discovering design opportunities, building prototypes, conducting usability testing, and optimizing iteratively, which would be more objective for revealing ergonomic problems.

\section{References}

[1] Wang Zhibao, Sun Tieshan and Li Guoping, J. (2013) Population Research. 01, 66-77.

[2] Huang Yuexin, J. (2017) Packaging Engineering. 38, 177-181.

[3] Xin Xiangyang, J. (2015) Decoration. 261, 58-62.

[4] Yu Shulin, M. (2005) Basic Industrial Engineering. (Beijing: Higher Education Press).

[5] Hu Fei, J. (2011) Art and Design 02, 133-136.

[6] He Huang, Minggang Yang and Taifeng Lv, J. (2018) International Journal of Industrial Ergonomics. 68, 211-221. 